\title{
High-power free-electron maser operated in a two-mode frequency-multiplying regime
}

\author{
N. Yu. Peskov, ${ }^{1}$ I. V. Bandurkin, ${ }^{1}$ A. K. Kaminsky, ${ }^{2}$ S. V. Kuzikov, ${ }^{1}$ E. A. Perelstein, ${ }^{2}$ \\ A. V. Savilov, ${ }^{1}$ S. N. Sedykh, ${ }^{2}$ and A. A. Vikharev ${ }^{1}$ \\ ${ }^{1}$ Institute of Applied Physics of the Russian Academy of Sciences, Nizhny Novgorod 603950, Russia \\ ${ }^{2}$ Joint Institute for Nuclear Research, Dubna 141980, Russia
}

(Received 29 March 2016; published 29 June 2016)

\begin{abstract}
The frequency multiplication effects in high-power free-electron masers (FEM) with Bragg cavities were studied to provide the advance of the oscillators into short-wavelength bands. Theoretical analysis of frequency-multiplying FEMs was carried out within the framework of the averaged coupled-wave approach. Proof-of-principle experiments were performed based on a moderately relativistic induction linac LIU-3000 (JINR). As a result, an FEM multiplier operated with a megawatt power level in the 6-mm and 4-mm wavelength bands at the second and third harmonics, respectively, was realized. The possibility of using two-mode bichromatic FEMs for powering a double-frequency accelerating structure was discussed.
\end{abstract}

DOI: 10.1103/PhysRevAccelBeams.19.060704

\section{INTRODUCTION}

Free-electron masers (FEMs) based on intense relativistic electron beams stay among the most powerful sources of pulsed microwave radiation. By now, in collaboration between the Joint Institute for Nuclear Research (JINR, Dubna) and the Institute of Applied Physics RAS (IAP RAS, Nizhny Novgorod), a high-power narrow-band FEM with Bragg resonator based on the induction linac LIU3000 (JINR) was realized in the $30-\mathrm{GHz}$ frequency band (30-GHz JINR-IAP FEM) [1,2]. The radiation parameters achieved in these experiments (output power, electron efficiency, stability of the narrow-band operation regime, etc.) allow JINR-IAP FEM to be used in several physical and engineering applications, in particular, to study the heating stress effect in high-gradient accelerating structures for the CLIC project (CERN) [3,4].

For advancement of JINR-IAP FEMs into the short part of millimeter and submillimeter wavelength bands, the use of the frequency multiplication effects is beneficial. Such regimes of the fundamental-frequency harmonic generation are effectively exploited in optical, infrared, and terahertz free-electron lasers [5-8]. An obvious advantage of the FEM multiplier is the possibility to obtain high-frequency radiation keeping the beam energy and other parameters of the electron-optical system unchanged. Moreover, specific electron mode selection mechanisms featured by these systems permit selective excitation of the operating highfrequency mode in a strongly oversized cavity without any

Published by the American Physical Society under the terms of the Creative Commons Attribution 3.0 License. Further distribution of this work must maintain attribution to the author $(s)$ and the published article's title, journal citation, and DOI. additional resonant system at these high frequencies. This paper is devoted to a theoretical study of the Bragg FEM multiplier schemes at the millimeter wavelengths and their experimental realization based on the LIU-3000 accelerator. Applicability of such bichromatic sources for the multifrequency acceleration problem is discussed.

\section{OPERATING PRINCIPLES AND SIMULATIONS OF THE FEM MULTIPLIERS}

The proposed scheme of an FEM multiplier with Bragg resonator is shown in Fig. 1. The FEM is driven by a magnetically guided pencil-like electron beam moving with axial velocity $v_{\|}$near the axis of a helical wiggler of period $d_{w}$ and oscillating with the bounce frequency $\Omega_{b}=2 \pi v_{\|} / d_{w}$. The beam electrons interact with forward-propagating circularly polarized low-frequency (LF) wave "1"

$$
\mathbf{E}_{1}=A_{+}(z) \hat{\mathbf{E}}_{1}(r) \exp \left(i \omega_{L F} t-i h_{L F}^{+} z\right)
$$

under the fundamental resonance

$$
\omega_{L F}-h_{L F}^{+} v_{\|}=\Omega_{b} .
$$

Selective excitation of this wave is ensured by the Bragg cavity, which provides narrow-band feedback with counterpropagating wave " 2 "

$$
\mathbf{E}_{2}=A_{-}(z) \hat{\mathbf{E}}_{2}(r) \exp \left(i \omega_{L F} t+i h_{L F}^{-} z\right)
$$

in the vicinity of the operating frequency $\omega_{L F}$ under the Bragg resonance condition [9]

$$
h_{L F}^{+}+h_{L F}^{-}=\bar{h} \text {, }
$$




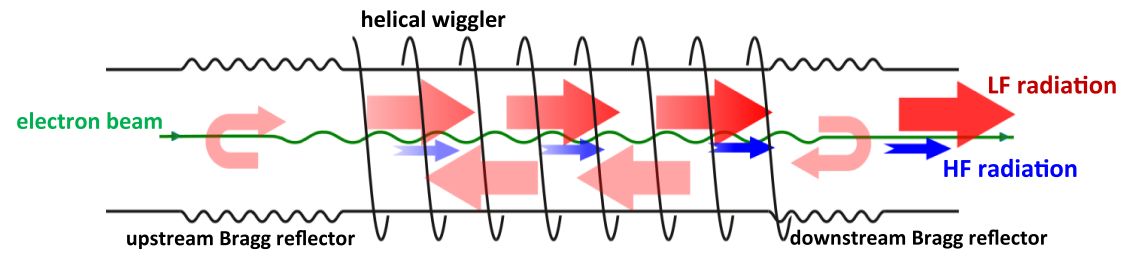

FIG. 1. Scheme of the FEM multiplier with a helical wiggler and a two-mirror Bragg resonator.

where $\bar{h}=2 \pi / d, d$ is the corrugation period of the Bragg structures and $\hat{\mathbf{E}}_{i}(r)$ are the functions describing the transverse structures of the corresponding modes of a circular waveguide.

Due to the nonlinearity of the electron bunching process during the e-beam/LF-wave interaction, the electron current contains high-frequency components at the harmonics of the fundamental frequency $\omega_{L F}$, which can be used for high-frequency (HF) generation. The sth harmonic of the electron current would excite HF wave "3"

$$
\mathbf{E}_{3}=A_{s}(z) \hat{\mathbf{E}}_{3}(r) \exp \left(i \omega_{H F} t-i h_{H F} z\right)
$$

at the frequency $\omega_{H F}=s \omega_{L F}$ under resonance with the $n$th harmonic of the bounce frequency

$$
\omega_{H F}-h_{H F} v_{\|}=n \Omega_{b} .
$$

Evidently, for the efficient LF and HF coexcitation by the same electron beam all the conditions (2), (4), and (6) should be fulfilled simultaneously. Figure 2 presents a dispersion diagram illustrating interaction of the beam electrons with LF and HF waves in the FEM multiplier.

The main advantage of the proposed scheme is relative simplicity of the FEM microwave system. Indeed, the electron bunching is provided by the LF wave, whereas at the high harmonic, a single-pass wave amplification takes place, which eliminates the necessity of the HF feedback. At the same time, this scheme has a certain electronic selectivity, since the axis-encircling electron beam efficiently interacts only with the circularly polarized waves having an azimuthal index equal to the corresponding bounce frequency harmonic $m=n$ [10]. Moreover, the frequency of HF oscillations is defined by the LF interaction, which allows additional discrimination of the parasitic HF waves with different radial indices. Finally, the operating current of the oscillator is defined by the starting current of the LF self-excitation, which is usually lower than the HF self-excitation current.

The steady-state operation regime of the considered Bragg FEM multiplier scheme can be described by the following equations:

$$
\frac{d A_{+}}{d z}-i \alpha(z) A_{-}=-i C_{L F} G_{1}
$$

$$
\begin{aligned}
\frac{d A_{-}}{d z}+i \alpha(z) A_{+} & =0, \\
\frac{d A_{s}}{d z} & =-i C_{H F} G_{s} .
\end{aligned}
$$

The first two equations [(7a) and (7b)] are written within the framework of the coupled-wave approach (cf. [9]), and describe mutual scattering of two counterpropagating waves, $A_{+}$and $A_{-}$, at the Bragg corrugation, as well as amplification of the synchronous wave, $A_{+}$, by the electron beam. The last equation [(7c)] is the excitation equation for the high frequency wave, $A_{s}$, generated by the $s$-harmonic of the electron current, $G_{s}$; the latter is given by Eq. (11) below (see [11] for details). In (7a)-(7c),

$$
\begin{aligned}
C_{L F} & =\frac{I_{0}}{I_{a}} \frac{\kappa_{\perp L F}^{+} \omega_{L F}}{N_{L F}^{+} h_{L F}^{+} c} \mathrm{~J}_{1}^{\prime}\left(\frac{\kappa_{\perp L F}^{+} K}{h_{w}}\right), \\
C_{H F} & =\frac{I_{0}}{I_{a}} \frac{\kappa_{\perp H F} \omega_{H F}}{N_{H F} h_{H F} c} \mathrm{~J}_{n}^{\prime}\left(\frac{\kappa_{\perp H F} K}{h_{w}}\right)
\end{aligned}
$$

are the gain (Pierce) parameters at low and high frequencies, respectively, $I_{0}$ is the beam current, $I_{a}=m c^{3} / e$, $h_{w}=2 \pi / d_{w}, h_{i}=\sqrt{\omega_{i}^{2} / c^{2}-\kappa_{\perp i}^{2}}$ and $\kappa_{\perp i}$ are the axial and transverse wave numbers of the corresponding waves, and $\alpha(z)$ is the Bragg coupling coefficient, which is equal to

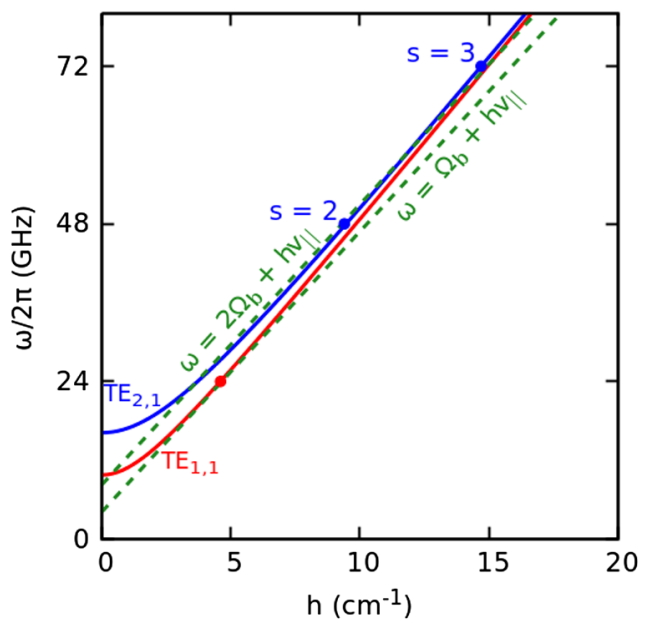

FIG. 2. Dispersion diagram illustrating the rf wave/e-beam interaction in the FEM multiplier. The solid lines correspond to the operating $\mathrm{TE}_{1,1}$ and $\mathrm{TE}_{2,1}$ modes and the dashed lines show the electron beam dispersion curves. 
$\alpha(z)=\alpha_{0}$ inside the Bragg reflectors, and to $\alpha(z)=0$ in the regular cavity section (scattering of HF waves at the Bragg corrugation is neglected). The electron-wave coupling coefficient $K$ in the regime of a reversed guide magnetic field $[12,13]$ is equal to (see [14] for details)

$$
K=\frac{\beta_{\perp}}{\beta_{\|}} \approx \frac{e H_{w}}{\gamma m c^{2}\left(\Omega_{b}+\omega_{H}\right)},
$$

where $\beta_{\perp, \|}$ are the corresponding normalized electron velocities, $\omega_{H}=e H_{0} / \gamma m c$ is the gyrofrequency of the electrons in the guide magnetic field $H_{0}$, and $H_{w}$ is the amplitude of the wiggler field. Normalization of the partial wave amplitudes corresponds to the following expression for the carried power:

$$
P_{i}=I_{a}^{2} N_{i} h_{i}\left|A_{i}\right|^{2} / 4 \omega_{i},
$$

where $N_{i}=\left(\kappa_{\perp}^{2} R_{0}^{2}-m_{i}^{2}\right) \mathrm{J}_{m_{i}}^{2}\left(\kappa_{\perp i} R_{0}\right) / 2$ are the norms of the partial waves, $m_{i}$ are their azimuthal indices, $\mathbf{J}_{m}$ and $\mathbf{J}_{m}^{\prime}$ are the corresponding Bessel functions and their derivatives, and $R_{0}$ is the waveguide radius.

The harmonics of the electron current

$$
G_{s}=(1 / \pi) \int_{0}^{2 \pi} K \exp (-i s \theta) d \theta_{0}
$$

can be found from the electron motion equations, which have the following form:

$$
\begin{aligned}
\frac{d \gamma}{d z}= & K\left[\kappa_{\perp L F}^{+} J_{1}^{\prime}\left(\frac{\kappa_{\perp L F}^{+} K}{h_{w}}\right) \operatorname{Im}\left(A_{+} e^{-i \theta}\right)\right. \\
& \left.+\sum_{s} \kappa_{\perp H F s} J_{n}^{\prime}\left(\frac{\kappa_{\perp H F s} K}{h_{w}}\right) \operatorname{Im}\left(A_{s} e^{-i \theta_{s}}\right)\right], \\
\frac{d \theta}{d z}= & h_{w}+h_{L F}^{+}-\frac{\omega_{L F} \gamma}{c p_{\|}},
\end{aligned}
$$

where $\theta=\omega_{L F} t-\left(h_{L F}^{+}+h_{w}\right) z$ and $\theta_{s}=s \theta+z\left[(n-s) h_{w}-\right.$ $\left.s h_{L F}^{+}+h_{H F}\right]$ are the electron phases to the LF and HF waves, respectively.

The boundary conditions for the partial waves in Eq. (7) correspond to the absence of the wave fluxes from outside the cavity,

$$
\left.A_{+}\right|_{z=0}=0,\left.\quad A_{s}\right|_{z=0}=0,\left.\quad A_{-}\right|_{z=0}=0,
$$

where $L$ is the total cavity length. The boundary conditions for the monoenergetic nonmodulated electron beam in Eq. (12) have the form

$$
\left.\gamma\right|_{z=0}=\gamma_{0},\left.\quad \theta\right|_{z=0}=\theta_{0} \in[0,2 \pi) .
$$

It should be noted that in the simulations the initial velocity spread was taken into account as follows: the initial transverse velocities were introduced into the beam from the interval $\left[0, \beta_{\perp \max }\right]$ and correspond to the presence of parasitic cyclotron oscillations of the beam electrons before their entrance into the wiggler.

The FEM multiplier was simulated with the parameters close to experimental conditions at the LIU-3000 accelerator (see Sec. III below). The operating transverse electron velocity in the wiggler amounted to $\beta_{\perp} \approx 0.2$ and the maximum initial parasitic transverse velocity reached $\beta_{\perp \max } \approx 0.07$. Analysis of the dispersion diagrams presented in Fig. 2 shows that under the experimental conditions a joint excitation of the $\mathrm{TE}_{1,1} \mathrm{LF}$ and $\mathrm{TE}_{2,1} \mathrm{HF}$ waves is possible. The LF wave is excited at fundamental resonance (2), whereas the HF wave can be excited at the second bounce-frequency harmonic $(n=2)$ and at the second $(s=2)$ or third $(s=3)$ harmonic of the LF frequency under resonance condition (6).
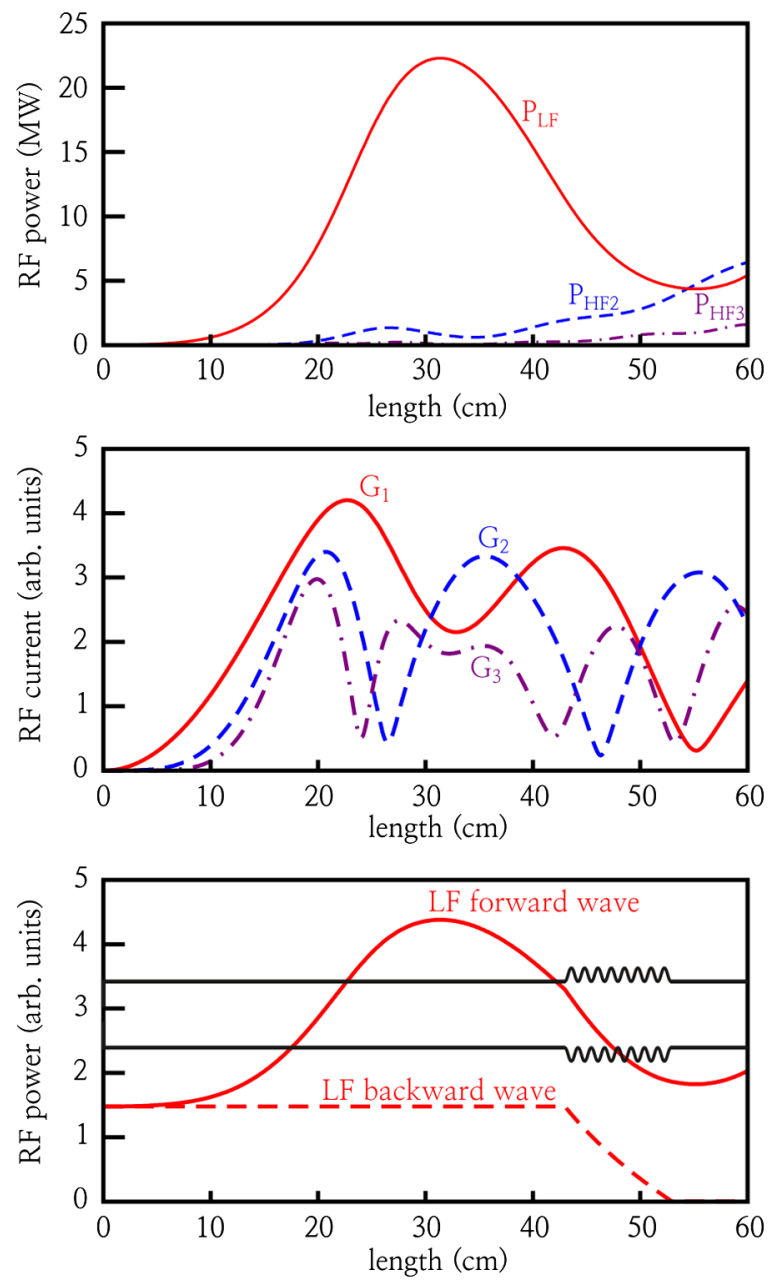

FIG. 3. The results of simulations of the FEM multiplier based on LIU-3000 within the framework of the averaged approach. Dependences on the axial coordinate $z$ of (a) the LF power $\left(P_{L F}\right)$ and HF powers $P_{H F 2,3}$ at the second $\left(P_{H F 2}\right)$ and third $\left(P_{H F 3}\right)$ harmonics, (b) amplitudes of the rf current harmonics $\left(G_{i}\right)$ and (c) amplitudes of the forward- and backward-propagating LF waves with respect to the position of the Bragg resonator. 
The FEM optimization was performed to obtain the maximum radiated power at a certain harmonic. Because of the nonlinearity of the beam bunching process, the cavity geometry should be optimized separately for each of the high harmonics (see Fig. 3). In the simulations carried out within the framework of the averaged approach described above, in the regular cavity section of $40 \mathrm{~cm}$ long and the output (downstream) mirror reflection coefficient $R_{\text {out }} \approx$ 0.65 the maximum radiated power at the second harmonic reached 7.1 MW (which corresponds to an electron efficiency of $4.2 \%$ ). The power of the third harmonic radiation at these parameters amounted to $1.4 \mathrm{MW}$. A decrease in the output reflection coefficient $R_{\text {out }} \approx 0.55$ leads to the enhancement of radiated power at the third harmonic up to $1.6 \mathrm{MW}$ (an efficiency of $\sim 0.9 \%$ ), but is accompanied with a drop in the second-harmonic radiation power down to $6.4 \mathrm{MW}$. For the sake of calculation simplicity, the input (upstream) Bragg reflector was replaced by a lumped mirror with the reflection coefficient $R_{i n}=1$. The efficiency of LF generation in these regimes was about $3 \%-5 \%$, which is much lower compared with the results of the previous experiments [1-4] and can be explained by the fact that the optimal beam parameters for the high-harmonic radiation are substantially different from those of the conventional single-frequency FEM regime. It should also be noted that for the chosen cavity geometry the radiation at harmonics higher than $s>3$ and $n>2$ was rather small.

\section{PROOF-OF-PRINCIPLES EXPERIMENTS IN THE V BAND}

The proposed scheme of a Bragg FEM multiplier was tested in a preliminary experiment based on a linear induction accelerator LIU-3000 0.8 MeV/200 A/250 ns. Inside the interaction region, the beam was transported and focused by a solenoid with an axial magnetic field of about $0.1-0.2 \mathrm{~T}$. The operating transverse oscillations of the beam electrons were pumped in a helical wiggler with a period of $6 \mathrm{~cm}$ and a transverse magnetic field amplitude of up to $0.15 \mathrm{~T}$. The regime of a reversed guide magnetic field $[12,13]$ was chosen for the FEM operation to reduce sensitivity to the initial spread of beam parameters and to provide a high quality of the helical electron beam formation [14,15].

The microwave system of the FEM represented a twomirror Bragg resonator consisting of two corrugated sections with a period of $8.65 \mathrm{~mm}$ and $23 \mathrm{~cm}$ long for the input (upstream) and 12 to $16 \mathrm{~cm}$ for the output (downstream) reflectors, respectively, which were separated by a regular section having a radius of $0.9 \mathrm{~cm}$ and 40 to $50 \mathrm{~cm}$ long [16]. This cavity ensured a feedback loop for the forward-propagating wave of $\mathrm{TE}_{1,1}$ type and a backward-propagating wave of $\mathrm{TM}_{1,1}$ type in the vicinity of the operating frequency $24 \mathrm{GHz}$ and provided excitation of the oscillator at the fundamental harmonic of bounce frequency under resonance condition (2). In accordance with Eq. (6), the $\mathrm{TE}_{2,1}$ wave should be excited at the second harmonic of the bounce frequency $n=2$ (see Fig. 2) in a frequency band of $48 \mathrm{GHz}$ (second harmonic of the rf current $s=2$ ) and $72 \mathrm{GHz}$ (third harmonic of the rf current $s=3$ ).

The results of the proof-of-principle experiments with a Bragg FEM multiplier are shown in Fig. 4. In the region of design parameters, two-wave oscillation at the fundamental bounce harmonic at a frequency of about $24 \mathrm{GHz}$ (operating $\mathrm{TE}_{1,1}$ wave) and at the second bounce harmonic at frequencies near 48 and $72 \mathrm{GHz}$ (which, according to simulations, corresponds to the excitation of the $\mathrm{TE}_{2,1}$ wave) was obtained. The radiation spectrum in the $\mathrm{V}$ band was measured by the calibrated set of cutoff waveguide filters having an accuracy of about $1 \mathrm{GHz}$ and demonstrated sharp resolved maxima at the frequencies given above. In the resonator with $40 \mathrm{~cm}$ long regular section and $16 \mathrm{~cm}$ long downstream reflector, the radiated power reached, according to the calorimetric measurements, $5 \mathrm{MW}$ at the fundamental harmonic and 1.5 MW at the
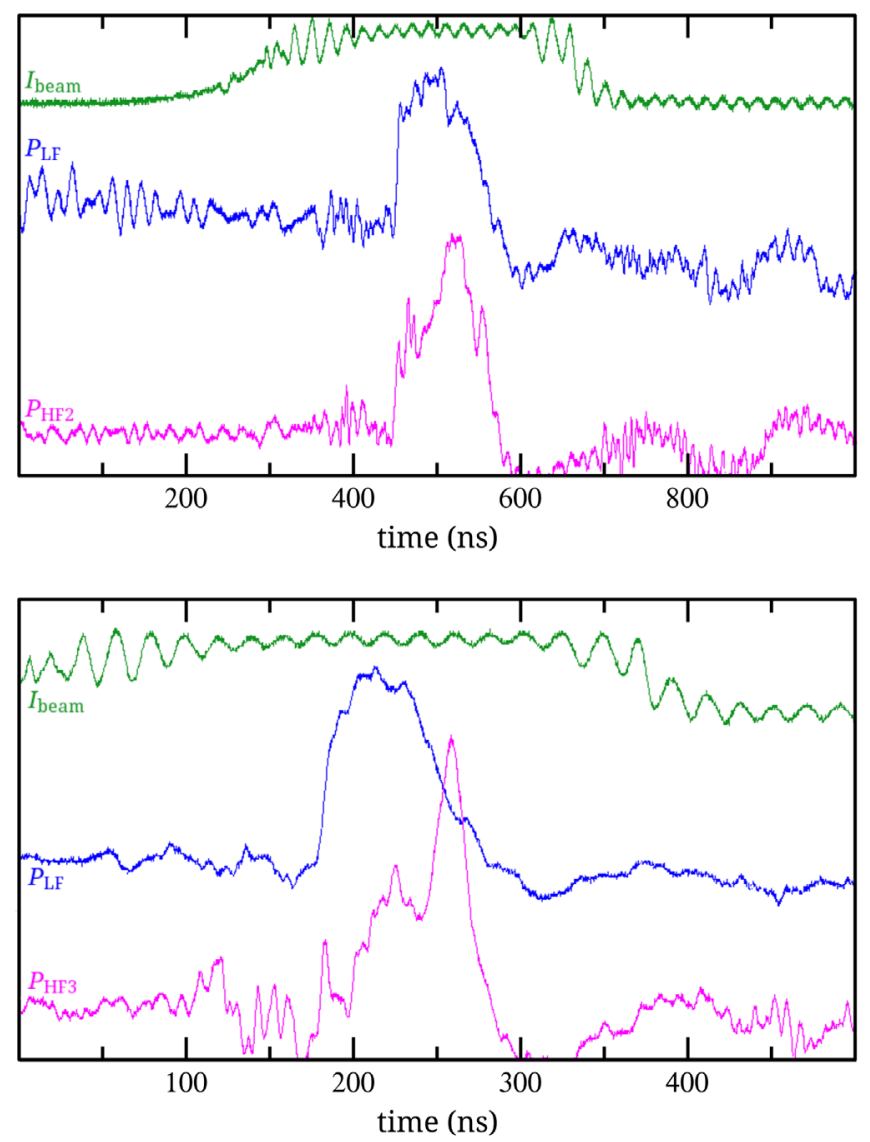

FIG. 4. The results of experimental studies of the FEM multiplier based on the LIU-3000 accelerator. Typical oscilloscope traces of the beam current $I_{\text {beam }}$ and the rf pulses in the twowave generation regime of the fundamental harmonic at $24 \mathrm{GHz}$ $\left(P_{L F}\right)$ with (a) the second harmonic at $48 \mathrm{GHz}\left(P_{H F 2}\right)$ and (b) the third harmonic at $72 \mathrm{GHz}\left(P_{H F 3}\right)$. 
second harmonic [Fig. 4(a)]. The third-harmonic radiation power in this case was about $100 \mathrm{~kW}$, which was estimated by measuring the detector signals. To increase radiated power at the third harmonic, the downstream reflector length was reduced down to $12 \mathrm{~cm}$, while the regular section was lengthened up to $50 \mathrm{~cm}$. As a result, the output power at the third harmonic reached the level of a few hundred kilowatts [Fig. 4(b)]. In accordance with the simulations prediction, this accompanied by deterioration of the second harmonic generation, which was observed at the level $\sim 1-1.2 \mathrm{MW}$ in this case.

Tuning of the FEM to the maximum output power at different harmonics also required changing the wiggler and guide fields. At the second harmonic, the optimal wiggler field was about $0.11-0.12 \mathrm{~T}$ with the guide field of about $0.15 \mathrm{~T}$. At the third harmonic, since the interaction occurs at the second bounce-frequency harmonic with the operating wave of the same type $\left(\mathrm{TE}_{2,1}\right)$ but higher frequency, this wave possesses higher group velocity and, according to resonance condition (6), requires lower particles transverse velocity. Thus, following to the simulations prediction, enhancement of the third-harmonic radiation power was obtained with decrease of the wiggler field down to $0.9-0.1 \mathrm{~T}$.

\section{THE USE OF A BICHROMATIC FEM MULTIPLIER FOR POWERING A DOUBLE- FREQUENCY ACCELERATING STRUCTURE}

Theoretical and experimental studies confirmed the possibility of using the frequency multiplication effects to advance moderately relativistic FEMs into the shortwavelength ranges. The FEM multiplier considered above can be used in a number of actual applications requiring high-power short-wavelength radiation. For this purpose, different frequency components in the radiated pulse can in principle be easily separated from each other because of the large frequency distance between them. Moreover, the coherent bichromatic radiation generated by an FEM multiplier is beneficial itself, in particular, for powering a double-frequency accelerating structure. Such a structure of the novel type was proposed in [17] and [18]. The addition of a high harmonic to the main accelerating field allows, first, to reach a higher accelerating gradient due to the reduction of the structure surface exposed to rf fields and the exposure time, as well as due to the reduction of those surface field components which are responsible for the autoelectron emission [17,18]. Second, the use of the higher harmonics can also reduce the consumed rf power for a given value of the accelerating gradient due to the enhancement of the structure's shunt impedance, as was shown in [19]. To feed these multifrequency structures, one needs high-power radiation which consists of several mutually phased harmonics. The mentioned requirement can, in principle, be satisfied using rf sources of different types. In particular, in [20], two independent rf sources at frequencies of 2.856 and $5.712 \mathrm{GHz}$, respectively, were considered to feed a doublemode accelerating structure in the experiment aimed to show the breakdown threshold increase. In particular, in that experiment it was assumed to use an S-band klystron of 15 to $20 \mathrm{MW}$ output power and a C-band gyromultiplier of 5 to $6 \mathrm{MW}$ output power. In this scheme the klystron generated a low-frequency high power itself and also was used for provision of an input power signal by means of a directional coupler for the mentioned gyromultiplier. In comparison with such a combined rf source, the FEM multiplier described above seems more attractive because the complex ratio of signals at two necessary frequencies is not correlated in this case with the possible instabilities of two different particle beams. The scheme of the planned experiment for testing the double-frequency accelerating structure by means of the developed FEM multiplier is shown in Fig. 5. This scheme is based on selective conversion of the rotating $\mathrm{TE}_{1,1}$ mode at $24 \mathrm{GHz}$ (marked by the red color) and $\mathrm{TE}_{2,1}$ mode at $48 \mathrm{GHz}$ (blue color) into fundamental $\mathrm{TE}_{1,0}$ modes of rectangular cross-section waveguides in the separated transmission lines. As in [20], the accelerating cavity operated with the superposition of $\mathrm{TM}_{0,1,0}$ (at the lowest frequency) and $\mathrm{TM}_{0,2,0}$ modes (at a double frequency) is to be fed by the mentioned transmission lines from two sides via the coupling holes.

Note that the lower-power FEM multiplier (5 MW at $24 \mathrm{GHz}$ and $1.5 \mathrm{MW}$ at $48 \mathrm{GHz}$ ) in comparison with the higher-power rf source described in [20] provides higher gradients in the accelerating structure, resulting in the assumption that the whole sizes should be scaled according to the frequency change. Indeed, the accelerating gradient $G$ can be written as [21]

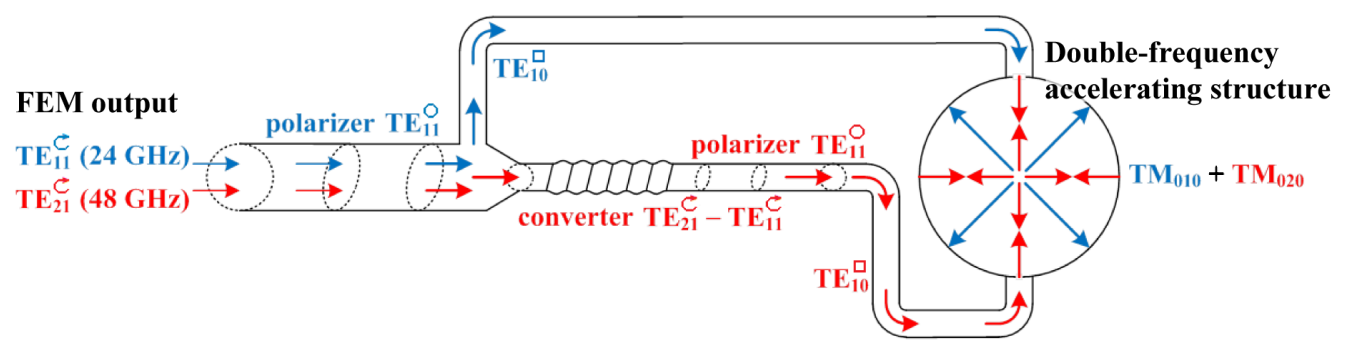

FIG. 5. Scheme of the planned experiment on testing a double-frequency accelerating structure by means of the FEM multiplier based on LIU-3000. 


$$
G=\sqrt{\frac{P \cdot R_{s h}}{L}},
$$

where $P$ is the radiated power, $R_{s h}$ is the shunt impedance per unit length and $L$ is the length of the structure. Taking into account that $R_{s h} \sim \omega^{1 / 2}$ and $L \sim \omega^{-1}$, we find that the accelerating gradient is scaled as $G \sim \omega^{3 / 4}$. Therefore, following Eq. (14), in the case of an FEM multiplier the gradient can be a factor of $\sim 2.4$ higher compared with the experiment proposed in [20], where a $300 \mathrm{MV} / \mathrm{m}$ surface field in the single-cell structure was calculated (the accelerating gradient is accordingly 2 times less). Thus, in the planned experiment with a FEM multiplier at 24 and $48 \mathrm{GHz}$ frequencies one can reach either a higher accelerating gradient or test a longer structure comprising many cells with the gradient kept unchanged.

\section{ACKNOWLEDGMENTS}

This work was partially supported by the Russian Science Foundation (Grant No. 14-19-01723).

[1] N. S. Ginzburg, A. A. Kaminsky, A. K. Kaminsky, N. Yu. Peskov, S. N. Sedykh, A. P. Sergeev, and A. S. Sergeev, High-Efficiency Single-Mode Free-Electron Maser Oscillator Based on a Bragg Resonator with Step of Phase of Corrugation, Phys. Rev. Lett. 84, 3574 (2000).

[2] A. K. Kaminsky, E. A. Perelstein, S. N. Sedykh, N. S. Ginzburg, S. V. Kuzikov, N. Yu. Peskov, and A. S. Sergeev, Demonstrating high-power $30-\mathrm{GHz}$ free-electron maser operation on a resonant load, Tech. Phys. Lett. 36, 211 (2010).

[3] N. S. Ginzburg, I. I. Golubev, A. K. Kaminsky, A.P. Kozlov, S. V. Kuzikov, E. A. Perelstein, N. Yu. Peskov, M. I. Petelin, S. N. Sedykh, A. P. Sergeev, A. S. Sergeev, A. A. Vikharev, and N. I. Zaitsev, Experiment on pulse heating and surface degradation of a copper cavity powered by powerful $30 \mathrm{GHz}$ free electron maser, Phys. Rev. ST Accel. Beams 14, 041002 (2011).

[4] V. G. Baev et al., Applied research using a $30 \mathrm{GHz}$ freeelectron maser: Experimental study of interacton of highpower pulsed radiation with metals, Radiophys. Quantum Electron. 54, 648 (2012).

[5] G. R. Neil, S. V. Benson, G. Biallas, J. Gubeli, K. Jordan, S. Myers, and M. D. Shinn, Second Harmonic FEL Oscillation, Phys. Rev. Lett. 87, 084801 (2001).

[6] L. H. Yu, L. DiMauro, A. Doyuran, W. S. Graves, E. D. Johnson, R. Heese, S. Krinsky, H. Loos, J. B. Murphy, G. Rakowsky, J. Rose, T. Shaftan, B. Sheehy, J. Skaritka, X. J. Wang, and Z. Wu, First Ultraviolet High-Gain HarmonicGeneration Free-Electron Laser, Phys. Rev. Lett. 91, 074801 (2003).

[7] G. DeNinno, E. Allaria, M. Coreno, S. Chowdhury, F. Curbis, M. B. Danailov, B. Diviacco, M. Ferianis,
E. Karantzoulis, E. C. Longhi, I. V. Pinayev, C. Spezzani, M. Trovò, and V. N. Litvinenko, Self-Induced Harmonic Generation in a Storage-Ring Free-Electron Laser, Phys. Rev. Lett. 100, 104801 (2008).

[8] V. V. Kubarev, G. N. Kulipanov, O. A. Shevchenko, and N. A. Vinokurov, in Proceedings of the 35th International Conference on Ir, Mm and THz Waves, Rome, Italy, 2010 (IEEE, New York, NY, 2010), p. Mo-F2.1.

[9] V. L. Bratman, G. G. Denisov, N. S. Ginzburg, and M. I. Petelin, FEL's with Bragg reflection resonators: Cyclotron autoresonance masers versus ubitrons, IEEE J. Quantum Electron. 19, 282 (1983).

[10] N. S. Ginzburg, N. Yu. Peskov, I. E. Kamenetsky, A. K. Kaminsky, A. A. Kaminsky, S. N. Sedykh, and A.P. Sergeev, Project of a large orbit FEM-oscillator operated at the second harmonic, Nucl. Instrum. Methods Phys. Res., Sect. A 429, 121 (1999).

[11] V. L. Bratman and A. V. Savilov, Competition of longitudinal modes and the scenario of single-mode regime build-up for the FOM-Fusion-FEM project, Nucl. Instrum. Methods Phys. Res., Sect. A 358, 182 (1995).

[12] A. A. Kaminsky, A. K. Kaminsky, and S. B. Rubin, V. P. Sarantsev, A.P. Sergeev, Experiments on the efficiency increase of FEL amplifier on the base of LIU-3000, Part. Accel. 33, 189 (1990).

[13] M.E. Conde and G. Bekefi, Experimental study of a 33.3- GHz free-electron-laser amplifier with a reversed axial guide magnetic field, Phys. Rev. Lett. 67, 3082 (1991).

[14] N. S. Ginzburg and N. Yu. Peskov, Nonlinear theory of a free electron laser with a helical wiggler and an axial guide magnetic field, Phys. Rev. ST Accel. Beams 16, 090701 (2013).

[15] N. S. Ginzburg, A. K. Kaminsky, A. A. Kaminsky, N. Yu. Peskov, S. N. Sedykh, A. P. Sergeev, and A. S. Sergeev, Theoretical and experimental comparison of FEL-oscillators with conventional and reversed guide field, IEEE Trans. Plasma Sci. 26, 536 (1998).

[16] I. V. Bandurkin, A. K. Kaminsky, E. A. Perelstein, N. Yu. Peskov, A. V. Savilov, and S. N. Sedykh, High-power freeelectron maser with frequency multiplication operating in a shortwave part of the millimeter wave range, Tech. Phys. Lett. 38, 759 (2012).

[17] S. V. Kuzikov, S. Yu. Kazakov, Y. Jiang, and J. L. Hirshfield, Asymmetric Bimodal Accelerator Cavity for Raising rf Breakdown Thresholds, Phys. Rev. Lett. 104, 214801 (2010).

[18] S. Yu. Kazakov, S. V. Kuzikov, Y. Jiang, and J. L. Hirshfield, High-gradient two-beam accelerator structure, Phys. Rev. ST Accel. Beams 13, 071303 (2010).

[19] S. G. Tantawi, Z. Li, and Ph. Borchard, United States Patent Application No. 20160014876, No. 14/795490, 2015, http:// www.freepatentsonline.com/y2016/0014876.html.

[20] Y. Jiang, S. V. Kuzikov, S. Yu. Kazakov, and J. L. Hirshfield, Multi-harmonic test setup for RF breakdown studies, Nucl. Instrum. Methods Phys. Res., Sect. A 657, 71 (2011).

[21] H. Wiedemann Particle Accelerator Physics, 3rd ed. (Springer, Berlin-Heidelberg, Germany, 2007). 